решетки. Саратов : Изд-во Сарат. ун-та, 1977. Т. 4. unary algebras. Mimeographed seminar notes. N. Y. : C. $28-40$.

U. C. Berkeley, 1967. 16 p.

7. Егорова Д. П. Структура конгруэнций унарной ал9. Карташов В. К. Независимые системы порождаюгебры // Упорядоченные множества и решетки. Саратов : Изд-во Сарат. ун-та, 1978. Т. 5. С. 11-44. щих и свойство Хопфа для унарных алгебр //Дискрет8. Johnson J., Seifert R. L. A survey of multi- $10.4213 /$ dm1027.

\title{
On Conditions for Distributivity or Modularity of Congruence Lattices of Commutative Unary Algebras
}

\begin{abstract}
V. K. Kartashov, A. V. Kartashova, V. N. Ponomarjov
Volgograd State Socio-pedagogical University, Russia, 400066, Volgograd, Lenina pr., 27, kartashovvk@yandex.ru, kartashovaan@yandex.ru

The paper is devoted to the problem of describing unary algebras whose congruence lattices have a given property. By now this problem has been solved for algebras with one unary operation. In the paper it is shown that this problem is much more difficult for arbitrary commutative unary algebras. We give some necessary conditions for such lattices to be distributive or modular. Besides, it is proved here that a lattice of all subsets of a set is isomorphic to the congruence lattice of a suitable connected commutative unary algebra.
\end{abstract}

Key words: commutative unary algebra, distributive lattice, modular lattice, congruence lattice of an algebra.

\section{References}

1. Gratzer G., Shmidt E. T. Characterizations of congruence lattices of abstract algebras. Acta Sci. Math., 1963, vol. 24, pp. 34-59.

2. Mal'tsev A. I. Algebraic Systems. Berlin, SpringerVerlag, 1976, 392 p. (Rus. ed. : Mal'tsev A. I. Algebraicheskie sistemy. Moscow, Nauka, 1970, 392 p.)

3. Skornjakov L. A. Unars. Coll. Math. Soc. J. Bolyai., 1982. vol. 29. Universal Algebra (Esztergom 1977). pp. $735-743$.

4. Skornjakov L. A. Complements in the lattice of congruences. Mathematics of the USSR-Sbornik, 1972. vol. 17, no 1. pp. 148-181. DOI: 10.1070/SM1972v017n 01ABEH001495.

5. Berman J. On the congruence lattices of unary algebras. Proc. Amer. Math. Soc., 1972. vol. 36. no 1. pp. $34-38$
6. Egorova D. P., Skornjakov L. A. O strukture kongrujencij unarnoj algebry [On congruence lattice of a unary algebra]. Uporjadochennye mnozhestva i reshetki [Ordered sets and lattices]. Saratov, Saratov Univ. Press, 1977, vol. 4. pp. 28-40 (in Russian).

7. Egorova D. P. Struktura kongrujencij unarnoj algebry [The congruence lattice of a unary algebra]. Uporjadochennye mnozhestva $i$ reshetki [Ordered sets and lattices]. Saratov, Saratov Univ. Press, 1978, vol. 5. pp. 11-44 (in Russian).

8. Johnson J., Seifert R. L. A survey of multi-unary algebras. Mimeographed seminar notes. New York, U. C. Berkeley, 1967, 16 p.

9. Kartashov V. K. Independent systems of generators and the Hopf property for unary algebras. Discrete Mathematics and Applications, 2008, vol. 18, iss. 6, pp. 625-630. DOI: 10.1515/DMA.2008.047.

УДК 512.567.5

\section{О РЕШЕТКАХ КОНГРУЭНЦИЙ ПРЯМЫХ СУММ СИЛЬНО СВЯЗНЫХ КОММУТАТИВНЫХ УНАРНЫХ АЛГЕБР}

\section{А. В. Карташова}

Кандидат фризико-математических наук, доцент кафредры алгебры, геометрии и математического анализа, Волгоградский государственный социально-педагогический университет, kartashovaan@yandex.ru

Объединение любого семейства попарно непересекающихся унарных алгебр называют их прямой суммой. Говорят, что унарная алгебра сильно связна, если она порождается любым своим элементом. В данной работе исследуется решетки конгруэнций коммутативных унарных алгебр с конечным числом операций, у которых каждая связная компонента является 
сильно связной. Найдено необходимое и достаточное условие, при котором решетка конгруэнций произвольной алгебры из этого класса является дистрибутивной. Описан также класс всех дистрибутивных решеток конгруэнций алгебр из обозначенного класса.

Ключевые слова: коммутативная унарная алгебра, сильно связная алгебра, решетка конгруэнций алгебры.

\section{ВВЕДЕНИЕ}

Унарной алгеброй называется алгебра, сигнатура которой состоит из унарных символов. Любую унарную алгебру, очевидно, можно рассматривать как автомат без выхода. Поэтому унарные алгебры привлекали внимание многих исследователей. Значительное место в этих исследованиях занимают решетки конгруэнций унарных алгебр, которые несут важную информацию о свойствах самих алгебр.

Исследования в этом направлении достаточно глубоко продвинуты для случая решеток конгруэнций унаров, т.е. алгебр с одной унарной операцией. В [1] описаны унары, решетка конгруэнций которых либо полумодулярна сверху, либо атомарна. В [2] и [3] найдены условия, при которых решетка конгруэнций унара является решеткой с дополнениями, дистрибутивной, модулярной, цепью, либо стоуновой решеткой.

Решетки конгруэнций унарных алгебр, сигнатура которых содержит более одной операции, изучены мало. Такие решетки рассматривались рядом авторов (см., например, [4-7]). Однако, к настоящему времени для них получено значительно меньше результатов.

Унарная алгебра $\langle A, \Omega\rangle$ называется коммутативной, если $f(g(a))=g(f(a))$ для любых $f, g \in \Omega$ и $a \in A$.

Объединение любого семейства попарно непересекающихся унарных алгебр называют их прямой суммой. В частности, запись $\mathfrak{A}=\mathfrak{A}_{1}+\mathfrak{A}_{2}$ означает, что алгебра $\mathfrak{A}$ является прямой суммой алгебр $\mathfrak{A}_{1}$ и $\mathfrak{A}_{2}$.

Унарная алгебра называется сильно связной, если она порождается любым своим элементом. Например, сильно связными унарами являются циклы и только они (циклом называют однопорожденный унар $\langle A, f\rangle$ такой, что $f^{n}(a)=a$, где $a$ - порождающий элемент этого унара, $n \in \mathbb{N}$ ).

Многообразие унарных алгебр конечной сигнатуры $\Omega$ называется сильно регулярным, если оно определяется тождествами вида $w(x)=x$, где слово $w$ содержит все символы из $\Omega$ (см, например, [8]).

Нетрудно показать, что каждая коммутативная связная алгебра любого сильно регулярного многообразия является сильно связной. К таким многообразиям относится, например, многообразие $\mathscr{A}_{1,1}$ алгебр с двумя унарными операциями $f$ и $g$, определяемое тождествами $f(g(x))=g(f(x))=x$ (см. [9-11])

В дальнейшем будем обозначать через $\mathfrak{K}$ класс всех коммутативных унарных алгебр с конечным числом операций, каждая из которых либо сильно связна, либо является прямой суммой сильно связных алгебр.

В данной работе найдено необходимое и достаточное условие, при котором решетка конгруэнций алгебры из класса $\mathfrak{K}$ дистрибутивна, и охарактеризован класс всех дистрибутивных решеток конгруэнций алгебр этого класса.

\section{1. ОСНОВНЫЕ ОПРЕДЕЛЕНИЯ И ВСПОМОГАТЕЛЬНЫЕ РЕЗУЛЬТАТЫ}

Пусть $\mathfrak{A}=\langle A, \Omega\rangle-$ произвольная унарная алгебра. Через $\Omega^{*}$ обозначается свободный моноид слов с порождающим множеством $\Omega$ относительно композиции. Единицей в $\Omega^{*}$ служит пустое слово $\emptyset$. В дальнейшем $\mathbb{N}$ всюду означает множество положительных целых чисел и $\mathbb{N}_{0}=\mathbb{N} \cup\{0\}$.

Результат $w(a)$ применения слова $w \in \Omega^{*}$ к элементу $a \in A$ определяется индукцией по длине слова $w$ (см. [12, с. 142]). Отсюда, если $w=w_{1} w_{2}$, то $w(a)=w_{1}\left(w_{2}(a)\right)$, где $w, w_{1}, w_{2} \in \Omega^{*}$ и $a \in A$. По определению также полагаем $f^{0}(a)=\emptyset a=a, f^{n}(a)=f\left(f^{n-1}(a)\right)$ для произвольных $f \in \Omega, a \in A$ и $n \in \mathbb{N}$. 
Для любого слова $u \in \Omega^{*}$ определим отображение $\delta_{u}: A \rightarrow A$ равенством $\delta_{u}(a)=u(a)$ для любого $a \in A$. Множество $\left\{\delta_{u} \mid u \in \Omega^{*}\right\}$ образует полугруппу $S(\mathfrak{A})$ относительно композиции. Эта полугруппа называется характеристической полугруппой унарной алгебрь 2 . В [13] показано, что характеристическая полугруппа всякой сильно связной коммутативной унарной алгебры является абелевой группой.

Для любого элемента $а$ произвольной унарной алгебры $\mathfrak{A}$ через $(a)$ обозначается подалгебра, порожденная элементом $a$.

Очевидно, что для любой коммутативной унарной алгебры $\mathfrak{A}=\langle A, \Omega\rangle$ бинарное отношение $\eta=\{(a, b) \in A \times A \mid(a) \cap(b) \neq \emptyset\}$ является конгруэнцией этой алгебры. Классы конгруэнции $\eta$ называются компонентами связности алгебры $\mathfrak{A}$. Легко проверить, что каждая компонента связности будет подалгеброй этой алгебры.

Коммутативная унарная алгебра называется связной, если она имеет ровно одну компоненту связности.

Лемма 1. Если унарная алгебра $\mathfrak{A}$ содержит более двух компонент связности, то решетка Соп конгруэнций этой алгебры не является дистрибутивной.

Доказательство аналогично доказательству леммы 1 из [3] для унаров.

Лемма 2. Пусть $\mathfrak{A}=\mathfrak{A}_{1}+\mathfrak{A}_{2}$, где $\mathfrak{A}_{1}=\left\langle A_{1}, \Omega\right\rangle$ и $\mathfrak{A}_{2}=\left\langle A_{2}, \Omega\right\rangle$ - сильно связные коммутативные унарные алгебры, причем для некоторой конгруэнции $\sigma \in$ Соп $\backslash\left\{1_{\text {сопя }}\right\}$ существуют элементь $a \in \mathfrak{A}_{1}, b \in \mathfrak{A}_{2}$, такие, что $(a, b) \in \sigma$. Тогда решетка СопА конгруэнций этой алгебры не является модулярной.

Доказательство. Положим $\tau=\left\{(x, y) \mid\left(x \sigma y \& x, y \in A_{1}\right) \vee x, y \in A_{2}\right\}, \gamma=\{(x, y) \mid \exists i \in\{1,2\}$ $\left.\left(x, y \in A_{i}\right)\right\}$. Непосредственная проверка показывает, что $\gamma, \tau \in C o n \mathfrak{A}$ и $\tau \leq \gamma$. Предположим теперь, что $\tau=\gamma$. Тогда для произвольного элемента $x \in A_{1}$ получаем $x \tau a$, поскольку $x \gamma a$ в силу определения конгруэнции $\gamma$, откуда $x \sigma a$. Это означает, что $A_{1} \subseteq[a]_{\sigma}$. Кроме того, так как алгебра $\mathfrak{A}_{2}$ сильно связная, то для любого $y \in A_{2}$ найдется слово $u \in \Omega^{*}$ такое, что $y=u(b)$. Отсюда $y \sigma u(a)$, поскольку $a \sigma b$ и, значит, $y \in[u(a)]_{\sigma}=[a]_{\sigma}$. Следовательно, $[a]_{\sigma}=A$, что противоречит условию. Таким образом, $\tau \neq \gamma$.

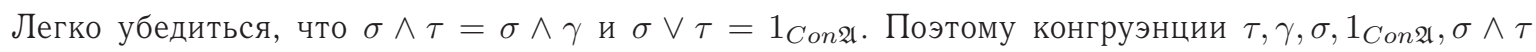
образуют пятиэлементную немодулярную решетку.

Для произвольной решетки $L$ через $L^{\prime}$ будем обозначать решетку, полученную из $L$ добавлением внешним образом наибольшего элемента.

Лемма 3. Пусть $\mathfrak{A}=\mathfrak{A}_{1}+\mathfrak{A}_{2}$, где $\mathfrak{A}_{1}=\left\langle A_{1}, \Omega\right\rangle$ и $\mathfrak{A}_{2}=\left\langle A_{2}, \Omega\right\rangle$ - сильно связнье коммутативные унарные алгебры, причем для любой конгруэнции $\theta \in$ Сопа и элементов $x \in A_{1}, y \in A_{2}$, справедлива импликация

$$
x \theta y \Rightarrow \theta=1_{\text {Con } \mathfrak{A}} \text {. }
$$

Тогда Cол $\mathfrak{A} \cong\left(\text { Con } \mathfrak{A}_{1} \times \operatorname{Con}_{2}\right)^{\prime}$.

Доказательство. Пусть $e$ - добавленный внешним образом наибольший элемент решетки $\left(\text { Con } \mathfrak{A}_{1} \times \operatorname{Con}_{2}\right)^{\prime}$. Для любой крнгруэнции $\theta \in C o n \mathfrak{A}$ положим

$$
\varphi(\theta)= \begin{cases}e, & \text { если } \theta=1_{\text {Соп }}, \\ \left(\theta_{1}, \theta_{2}\right), & \text { если } \theta \neq 1_{\text {Соn } \mathfrak{A}}\end{cases}
$$

где $\theta_{1}=\left\{(x, y) \mid x \theta y \& x, y \in A_{1}\right\}, \theta_{2}=\left\{(x, y) \mid x \theta y \& x, y \in A_{2}\right\}$.

Очевидно, что $\theta_{1} \in \operatorname{Con}_{1}, \theta_{2} \in \operatorname{ConA}_{2}$, причем $\theta \leq \rho \Rightarrow \varphi(\theta) \leq \varphi(\rho)$ для любых конгруэнций $\theta, \rho \in$ Con $\mathfrak{A}$.

Пусть теперь $\theta, \rho \in$ Con $\mathfrak{A} \backslash\left\{1_{\text {Con }}\right\}, \varphi(\theta) \leq \varphi(\rho)$ и $x \theta y$, где $x, y \in \mathfrak{A}$. Тогда $\{x, y\} \subseteq A_{i}$ для некоторого $i \in\{1,2\}$, откуда $(x, y) \in \theta_{1} \cup \theta_{2}$ и, значит, $(x, y) \in \rho_{1} \cup \rho_{2}$. Поэтому $(x, y) \in \rho$ и, следовательно, $\theta \leq \rho$. 
Убедимся, что отображение $\varphi: C o n \mathfrak{A} \rightarrow\left(\operatorname{Con} \mathfrak{A}_{1} \times \operatorname{ConA}_{2}\right)^{\prime}$ сюрьективно. Действительно, пусть $\left(\theta_{1}, \theta_{2}\right) \in \operatorname{ConA}_{1} \times$ Con: $_{2}$. Зададим отношение $\theta$ на множестве $A_{1} \cup A_{2}$ по правилу $\theta=\theta_{1} \cup \theta_{2} \cup\left\{(a, a) \mid a \in A_{1} \cup A_{2}\right\}$. Непосредственная проверка показывает, что $\theta \in \operatorname{Con} \mathfrak{A}$ и $\varphi(\theta)=\left(\theta_{1}, \theta_{2}\right)$.

Для любого числа $n \in \mathbb{N}$ через $\mathscr{L}_{n}$ будем обозначать решетку целых положительных делителей числа $n$. Через $\mathscr{N}$ обозначается решетка, двойственная к решетке целых положительных чисел по делимости.

Лемма 4. Пусть $\mathfrak{A}=\langle A, \Omega\rangle$ - сильно связная коммутативная унарная алгебра с конечным числом операций, решетка конгруэнций которой дистрибутивна. Тогда справедливы следующие утверждения:

1) если алгебра $\mathfrak{A}$ конечна, то Соп $\mathfrak{A} \cong \mathscr{L}_{n}$, где $n=|A|$;

2) если алгебра $\mathfrak{A}$ бесконечна, то Соп $\mathfrak{A} \cong \mathscr{N}$.

Доказательство. Заметим сначала, что решетка $S u b S(\mathfrak{A})$ подгрупп группы $S(\mathfrak{A})$ дистрибутивна, так как $S u b S(\mathfrak{A}) \cong C$ on $\mathfrak{A}$, ввиду [8, теорема 1]. Эта группа является конечно порожденной, поскольку $\mathfrak{A}$ - алгебра с конечным числом операций. Следовательно, $S(\mathfrak{A})$ - циклическая группа по теореме Ope (см. [14, теорема 78.2]).

Кроме того, $|S(\mathfrak{A})|=|\mathfrak{A}|$. Поэтому, если алгебра $\mathfrak{A}$ конечна, то $S u b S(\mathfrak{A}) \cong \mathscr{L}_{n}$, где $n=|S(\mathfrak{A})|=|A|$. Если же алгебра $\mathfrak{A}$ бесконечна, то $\operatorname{Con} \mathfrak{A} \cong \mathscr{N}$.

Лемма 5. Пусть $\mathfrak{A}_{1}=\left\langle A_{1}, \Omega\right\rangle$ и $\mathfrak{A}_{2}=\left\langle A_{2}, \Omega\right\rangle-$ сильно связные коммутативные унарные алгебры, $\mathfrak{A}=\mathfrak{A}_{1}+\mathfrak{A}_{2}, \theta \in$ Con $\mathfrak{A}, a \in A_{2} u A_{1} \subseteq[a]_{\theta}$. Тогда $\theta=1_{\text {Con }}$.

Доказательство. Пусть $b \in A_{2}$. Убедимся, что $b \in[a]_{\theta}$. Для этого зафиксируем произвольный элемент $c \in A_{1}$. Тогда $a \theta c$, так как $A_{1} \subseteq[a]_{\theta}$.

Кроме того, $b=u(a)$ для некоторого слова $u \in \Omega^{*}$, поскольку $\mathfrak{A}_{2}-$ сильно связная алгебра. Следовательно, $b \theta u(c)$, откуда $a \theta b$, так как $u(c) \in A_{1}$.

\section{2. ОСНОВНЫЕ РЕЗУЛЬТАТЫ}

Из лемм 2-4 непосредственно вытекает

Теорема 1. Решетка конгруэниий алгебры $\mathfrak{A}$ класса $\mathfrak{K}$ дистрибутивна тогда и только тогда, когда справедливо одно из следующих условий

1) $\mathfrak{A}$ сильно связная алгебра и $S(\mathfrak{A})$ - цүиклическая группа;

2) $\mathfrak{A}=\mathfrak{A}_{1}+\mathfrak{A}_{2}$, где $\mathfrak{A}_{1}, \mathfrak{A}_{2}-$ сильно связные алгебры, $S\left(\mathfrak{A}_{1}\right), S\left(\mathfrak{A}_{2}\right)$ - циклические группы, причем справедлива импликация

$$
(a, b) \in \theta \Rightarrow \theta=1_{C o n \mathfrak{A}}
$$

для любой конгруэнции $\theta \in$ Соп $\mathfrak{A}$ и элементов $a \in \mathfrak{A}_{1}, b \in \mathfrak{A}_{2}$.

Для любых целых чисел $s, k_{1}, k_{2}, \ldots, k_{s}$ обозначим через $\mathfrak{Z}\left(k_{1}, k_{2}, \ldots, k_{s}\right)$ алгебру $\left\langle\mathbb{Z}, f_{1}, f_{2}, \ldots, f_{s}\right\rangle$, где $\mathbb{Z}-$ множество целых чисел и $f_{i}(x)=x+k_{i}$ при всех $x \in \mathbb{Z}, i \in\{1,2, \ldots, s\}$.

Через $\mathfrak{Z}_{n}\left(k_{1}, k_{2}, \ldots, k_{s}\right), n \in \mathbb{N}$, обозначим алгебру $\left\langle\mathbb{Z}_{n}, f_{1}, f_{2}, \ldots, f_{s}\right\rangle$, где $\mathbb{Z}_{n}-$ множество классов вычетов по модулю $n, f_{i}(\bar{x})=\bar{x}+\bar{k}_{i}$ при всех $\bar{x} \in \mathbb{Z}_{n}, i \in\{1,2, \ldots, s\}$.

Теорема 2. Пусть $L$ - произвольная дистрибутивная решетка. Тогда $L \cong$ СопА для некоторой алгебры $\mathfrak{A} \in \mathfrak{K}$ тогда и только тогда, когда L изоморфна одной из решеток следующих видов:

1) $\mathscr{L}_{n}, n \in \mathbb{N}$;

2) $\mathscr{N}$;

3) $\mathscr{L}_{n}^{\prime}, n \in \mathbb{N}$;

4) $\left(\mathscr{L}_{n} \times \mathscr{N}\right)^{\prime}, n \in \mathbb{N}$;

5) $(\mathscr{N} \times \mathscr{N})^{\prime}$. 
Доказательство. Необходимость. Поскольку решетка конгруэнций алгебры $\mathfrak{A}$ дистрибутивна, то по лемме 1 алгебра $\mathfrak{A}$ содержит не более двух компонент связности. Если при этом алгебра $\mathfrak{A}$ является связной, то либо $C o n \mathfrak{A} \cong \mathscr{L}_{n}, n \in \mathbb{N}$, либо $C$ оп $\mathfrak{A} \cong \mathscr{N}$ по лемме 4 .

Пусть теперь алгебра $\mathfrak{A}$ состоит из двух компонент связности. Тогда $\mathfrak{A}=\mathfrak{A}_{1}+\mathfrak{A}_{2}$, где $\mathfrak{A}_{1}, \mathfrak{A}_{2}-$ некоторые коммутативные сильно связные алгебры. Кроме того, Con $\mathfrak{A} \cong\left(\operatorname{Con}_{1} \times \operatorname{Con}_{2}\right)^{\prime}$ в силу лемм 2 и 3.

Далее, решетки Con $\mathfrak{A}_{1}$ и Con $\mathfrak{A}_{2}$ также дистрибутивны, так как $\mathfrak{A}_{1}$ и $\mathfrak{A}_{2}-$ подалгебры алгебры $\mathfrak{A}_{2}$. Отсюда, снова применяя лемму 4 , получаем, что каждая из решеток $\operatorname{Con} \mathfrak{A}_{1}$ и $C$ оп $\mathfrak{A}_{2}$ либо изоморфна решетке $\mathscr{N}$, либо - одной из решеток вида $\mathscr{L}_{n}, n \in \mathbb{N}$. Осталось воспользоваться тем очевидным фактом, что для любых $n, m \in \mathbb{N}$ декартово произведение $\mathscr{L}_{n} \times \mathscr{L}_{m} \cong \mathscr{L}_{s}$, где $s \in \mathbb{N}$.

Достаточность. Заметим сначала, что $\mathfrak{Z}_{n}(1), \mathfrak{Z}(1,-1), n \in \mathbb{N}-$ сильно связные алгебры. Кроме того, $\operatorname{Con}_{n}(1) \cong \mathscr{L}_{n}$ ввиду [1, лемма 2] и $\operatorname{Con} \mathfrak{Z}(1,-1) \cong \mathscr{N}$ согласно [11, следствие 2 из леммы 3].

Пусть $n \in \mathbb{N}, \mathfrak{A}=\mathfrak{Z}_{n}(1)+\mathfrak{E}$, где $\mathfrak{E}=\left\langle\{e\}, f_{1}\right\}$ - одноэлементная алгебра. Тогда, очевидно, $\mathfrak{A} \in \mathfrak{K}$. Покажем, что $\operatorname{Con} \mathfrak{A} \cong \mathscr{L}_{n}^{\prime}$. Действительно, если $\bar{a} \theta$ для некоторой конгруэнции $\theta \in C$ Со $\mathfrak{A}$ и элемента

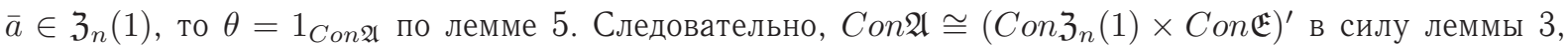
откуда, очевидно, Соп $\mathfrak{A} \cong \mathscr{L}_{n}^{\prime}$.

Пусть теперь $\mathfrak{A}=\mathfrak{Z}_{n}(1,1,1)+\mathfrak{Z}(0,1,-1)$. Тогда $\mathfrak{A} \in \mathfrak{K}$, так как $\mathfrak{Z}_{n}(1,1,1)$ и $\mathfrak{Z}(0,1,-1)-$ сильно связные коммутативные унарные алгебры.

Если при этом $\bar{a} \theta b$ для некоторых $\theta \in C o n \mathfrak{A}, \bar{a} \in \mathfrak{Z}_{n}(1,1,1), b \in \mathfrak{Z}(0,1,-1)$, то $f_{1}^{r}(\bar{a}) \theta f_{1}^{r}(b)$, т. е.

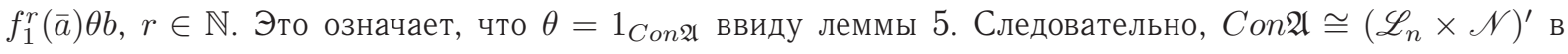
силу лемм 3 и 4.

Пусть, наконец, $\mathfrak{A}=\mathfrak{Z}(1,-1,0)+\mathfrak{Z}(0,1,-1)$ и $\theta \in C$ сол $\mathfrak{A}$ Предположив, что $a \theta b, a \in \mathfrak{Z}(1,-1,0)$, $b \in \mathfrak{Z}(0,1,-1)$, то $f_{1}^{r}(a) \theta f_{1}^{r}(b)$, откуда $f_{1}^{r}(a) \theta b$ и $f_{1}^{r}(a) \theta a$ для любого $r \in \mathbb{N}$.

Если же $c \in \mathfrak{Z}(1,-1,0)$ и $c=f_{2}^{s}(a)$, где $c \in \mathbb{N}$, то имеем $f_{2}^{s}(a) \theta f_{2} f_{1}(a)$, т. е. $c \theta a, c \theta b$. Следовательно, $\mathfrak{Z}(1,-1,0) \subseteq[b]_{\theta}$. Отсюда, снова применяя лемму 5 , получаем $\theta=1_{\text {Сопя }}$. Поэтому $\operatorname{Con} \mathfrak{A} \cong(\mathfrak{Z}(1,-1,0) \times \mathfrak{Z}(0,1,-1))^{\prime}$ в силу леммы 3 . Таким образом, $C$ on $\mathfrak{A} \cong(\mathscr{N} \times \mathscr{N})^{\prime}$ по лемме 4 .

\section{Библиографический список}

1. Berman J. On the congruence lattices of unary algebras // Proc. Amer. Math. Soc. 1972. Vol. 36, № 1. P. 34-38.

2. Егорова Д. П., Скорняков Л. А. О структуре конгруэнций унарной алгебры // Упорядоченные множества и решетки : сб. науч. тр. Саратов : Изд-во Сарат. ун-та, 1977. Вып. 4. С. 28-40.

3. Егорова Д. П. Структура конгруэнций унарной алгебры // Упорядоченные множества и решетки : сб. науч. тр. Саратов : Изд-во Сарат. ун-та, 1978. Вып. 5. C. $11-44$.

4. Gratzer G., Shmidt E. T. Characterizations of congruence lattices of abstract algebras // Acta Sci. Math. 1963. Vol. 24. P. 34-59.

5. Johnson J., Seifert R. L. A survey of multiunary algebras. Mimeographed seminar notes. N. Y. : U. C. Berkeley, 1967. 16 p.

6. Esik Z., Imreh B. Subdirectly irreducible commutative automata // Acta Cybernetica. 1981. Vol. 5, № 3. P. 251260.

7. Карташова A. B. О конечных решетках топо- логий коммутативных унарных алгебр // Дискретная математика. 2009. Т. 21, № 3. C. 119-132. DOI: $10.4213 / \mathrm{dm} 1065$.

8. Карташов В. К. Независимые системы элементов в коммутативных унарных алгебрах // Алгебра и теория чисел : современные проблемы и приложения : тез. докл. междунар. науч. конф. Саратов : Изд-во Сарат. ун-та, 2011. С. 29

9. Акатаев А. А., Смирнов Д. М. Решетки подмногообразий многообразий алгебр // Алгебра и логика. 1968. T. 7, № 1. C. 5-25. DOI: 10.1007/BF02218747.

10. Карташов B. K. О решетках квазимногообразий унаров // Сиб. мат. журн. 1985. Т. 26, № 3. С. 49-62. DOI: $10.1007 / \mathrm{BF} 00968621$.

11. Бощенко А. П. Решетки конгруэнций унарных алгебр с двумя операциями $f$ и $g$, удовлетворяющими тождествам $f(g(x))=g(f(x))=x$ или $f(g(x))=x /$ Волгоградский государственный педагогический университет. Волгоград, 1998. Деп. в ВИНИТИ 20.04.1998, № $1220-$ B98.

12. Мальцев А. И. Алгебраические системы. М. : Наука, 1970. 392 с. 
13. Esik Z., Imreh B. Remarks on finite commutative automata // Acta Cybernetica. 1981. Vol. 5, № 3. P. 143146.

\section{On Congruence Lattices of Direct Sums of Strongly Connected Commutative Unary Algebras}

\section{A. V. Kartashova}

Volgograd State Socio-Pedagogical University, Russia, 400066, Volgograd, Lenina prospekt., 27, kartashovaan@yandex.ru

A union of mutually disjoint unary algebras is called their direct sum. A unary algebra is said to be strongly connected if it is generated by its arbitrary element. In the present paper we investigate congruence lattices of the class of all algebras with finitely many operations whose every connected component is strongly connected. We give a necessary and sufficient condition for an algebra from this class to have a distributive congruence lattice (Theorem 1). Besides, all distributive congruence lattices of algebras from the above class are discribed (Theorem 2).

Key words: commutative unary algebra, strongly connected algebra, congruence lattice of an algebra.

\section{References}

1. Berman J. On the congruence lattices of unary algebras. Proc. Amer. Math. Soc., 1972, vol. 36, no 1, pp. 34-38.

2. Egorova D. P., Skornjakov L. A. O strukture kongrujencij unarnoj algebry [On congruence lattice of a unary algebra]. Uporjadochennye mnozhestva $i$ reshetki [Ordered sets and lattices]. Saratov, Saratov Univ. Press, 1977, vol. 4, pp. 28-40 (in Russian).

3. Egorova D. P. Struktura kongrujencij unarnoj algebry [The congruence lattice of a unary algebra]. Uporjadochennye mnozhestva $i$ reshetki [Ordered sets and lattices]. Saratov, Saratov Univ. Press, 1978, vol. 5, pp. 11-44 (in Russian).

4. Gratzer G., Shmidt E. T. Characterizations of congruence lattices of abstract algebras. Acta Sci. Math., 1963, vol. 24, pp. 34-59.

5. Johnson J., Seifert R. L. A survey of multi-unary algebras. Mimeographed seminar notes. New York, U. C. Berkeley, 1967. 16 p.

6. Esik Z., Imreh B. Subdirectly irreducible commutative automata // Acta Cybernetica, 1981, vol. 5, no 3, pp. 251260.

7. Kartashova A. V. On finite lattices of topologies of commutative unary algebras. Discrete Mathematics and Applications, 2009, vol. 19, iss. 4, pp. 431-443. DOI: 10.1515/DMA.2009.030.

8. Kartashov V. K. Nezavisimye sistemy jelementov v kommutativnyh unarnyh algebrah [Independent systems of elements in commutative unary algebras]. Algebra $i$ teorija chisel: sovremennye problemy $i$ prilozhenija : tezisy dokl. mezhdunar. nauch. konf., Saratov, 2011, pp. 29 (in Russian).

9. Akataev A. A., Smirnov D. M. Lattices of submanifolds in manifolds of algebras. Algebra and Logic, 1968, vol. 7, iss. 1, pp.2-13. DOI: 10.1007/BF02218747.

10. Kartashov V. K. Lattices of quasivarieties of unars. Siberian Mathematical Journal, 1985, vol. 26, iss. 3, pp. 346-357. DOI: 10.1007/BF00968621.

11. Boschenko A. P. Reshetki kongrujencij unarnyh algebr s dvumja operacijami $f$ i $g$, udovletvorjajushimi tozhdestvam $f(g(x))=g(f(x))=x$ ili $f(g(x))=x$ [Congruence lattices of unary algebras with two operations $f$ and $g$ which satisfy the identities $f(g(x))=g(f(x))=x$ or $f(g(x))=x]$. Volgograd pedagogical university. Volgograd, 1998. Dep. VINITI 20.04.1998, № 1220-B98 (in Russian).

12. Mal'tsev A. I. Algebraic Systems. Berlin, SpringerVerlag, 1976, 392 p. (Rus. ed. : Mal'tsev A. I. Algebraicheskie sistemy. Moscow, Nauka, 1970, 392 p.)

13. Esik Z., Imreh B. Remarks on finite commutative automata. Acta Cybernetica, 1981, vol. 5, iss. 3, pp. 143146.

14. Fuchs L. Abelian groups. Budapest, Publ. House of the Hungar. Acad. Sci., 1958, 367 p. 